\title{
Perspectives
}

\section{Standard port placement for laparoscopic pancreatic surgery: How we do it}

\author{
Jitendra H Mistry $^{1 *}$, Sanjiv Haribhakti ${ }^{2}$ \\ ${ }^{1}$ Baroda Laparoscopy Hospital, Institute of Gastroenterology \& Research Center, Vadodara, Gujarat, India \\ ${ }^{2}$ Kaizen Hospital, Institute of Gastroenterology \& Research Center, Ahmedabad, Gujarat, India \\ *Correspondence: jitlap@gmail.com
}

Abstract: Advanced laparoscopic surgeries are technically demanding, among which laparoscopic pancreatic surgeries are the most challenging. One of the technical difficulties is the improper port placement. As there is no standard port placement for laparoscopic pancreatic surgeries, it is difficult to get proper angle to work at different steps of the surgery if ports are not well placed. It is also responsible for musculoskeletal problems to the operating surgeon. Here we propose a standard port placement for laparoscopic pancreatic surgery which gives ergonomic comfort in all laparoscopic pancreatic surgeries.

Keywords: Advanced laparoscopic surgery; pancreatic surgery; laparoscopic surgery

Received: $5^{\text {th }}$ Sep. $2017 \quad$ Accepted: $15^{\text {th }}$ Nov. $2017 \quad$ Online: $25^{\text {th }}$ Nov. 2017

\section{Introduction}

Technical difficulty is the most important reason why advanced laparoscopic surgery has lagged behind basic laparoscopic surgery. Among the advanced laparoscopic procedures, laparoscopic pancreatic surgery is the most challenging. It has become unpopular due to various reasons such as technically demanding, long duration of dissection around the major vessels, and complexity of the reconstruction ${ }^{[1]}$. Some of the major reasons for technical difficulties are due to improper port placement and inability to find proper angle to work on. Those are also the most common reasons for having strains during laparoscopic surgery. For a majority of the pancreatic procedures, port placement has not been standardised. The aim of this article is to propose a standard port placement for laparoscopic pancreatic surgery which provides ergonomic convenience in all pancreatic surgeries.

\section{Conventional principles of port placement}

1. Straight line principle of laparoscopy (Figure 1a)

The surgeon, the target area of surgery and the monitor must be in a straight line. The level of the monitor must be at the level of surgeon's eyes.

2. 'Baseball diamond' concept of laparoscopy (Figure 1b)

This principle provides triangulation of the instruments and avoids sword-fighting between the instruments.

3. Manipulation angle (Figure 1c)

Manipulation angle is the angle between two working ports. The ideal manipulation angle is $60^{\mathrm{o}[2]}$.

4. Azimuth angle (Figure 1c)

Azimuth angle is the angle between one instrument and the optical axis of the endoscope. The ideal azimuth angle is equal for both the active and assisting instruments ${ }^{[2]}$.

5. Elevation angle (Figure 1c)

Elevation angle is the angle between the instrument and the horizontal plane. The ideal elevation angle is $60^{\circ{ }^{[2]}}$.

These angles determine the optimal port placement in a particular laparoscopic surgery. In basic laparoscopic procedures such as appendectomy or cholecystectomy, these angles remain fixed as the site of surgery remains fixed at one place. 


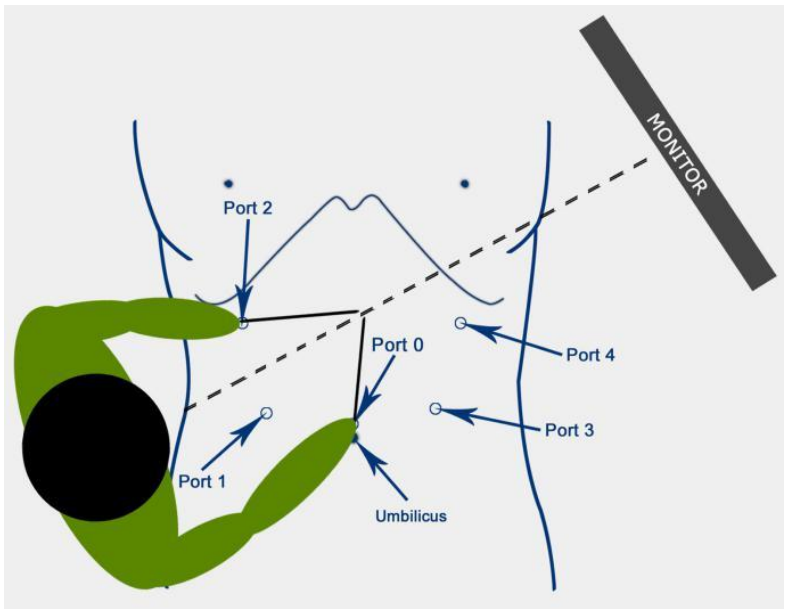

Figure 1a. Straight line principle of laparoscopy

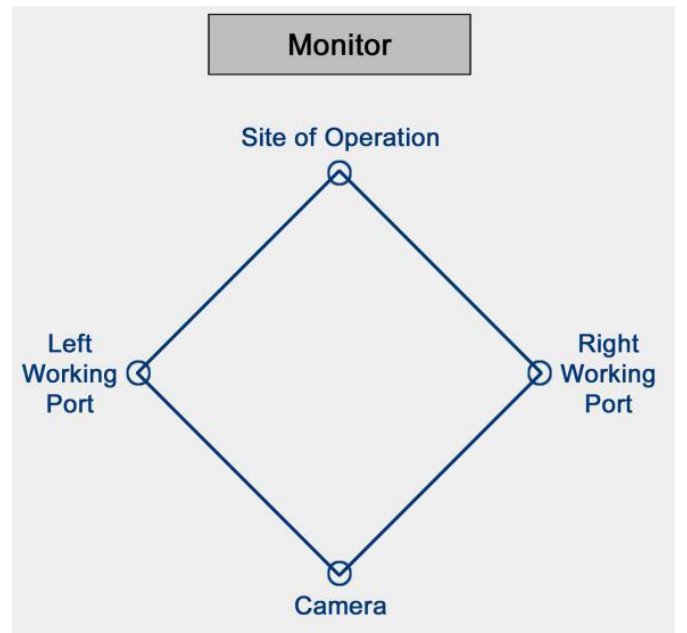

Figure 1b. "Baseball diamond" concept of laparoscopy

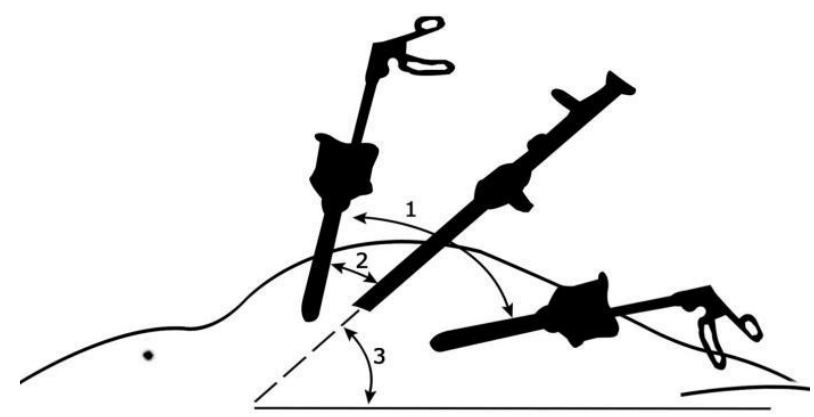

Figure 1c. Manipulation angle (1 \& 2) and Azimuth angle (3)

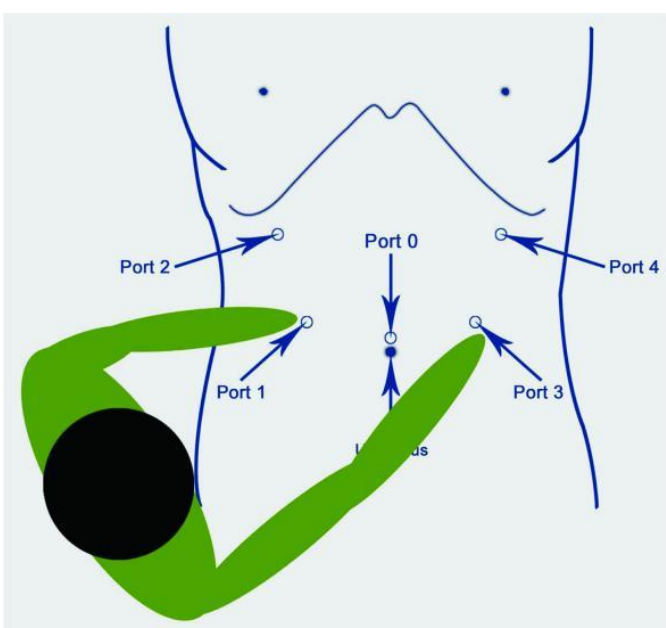

Figure 2a. Ergonomically less comfortable position
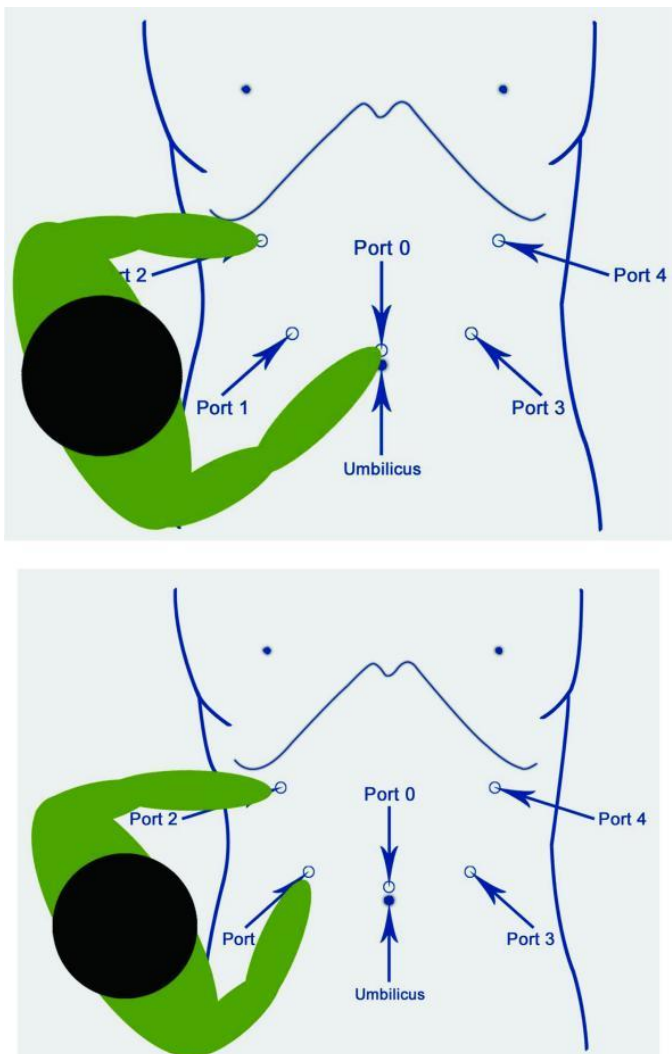

Figure 2b. and 2c. Ergonomically more comfortable position

In laparoscopic pancreatic surgery, as the point and angle of surgery vary from step to step, it requires extremely precise and calculated port placement which maintains the above-mentioned principles at every step and allows shuffling of the instruments to get the appropriate vision and angle to work.

\section{Hurdles while performing laparoscopic pancreatic surgery}

Pancreas is a retroperitoneal organ situated across the abdomen with oblique axis. It is not possible to deal with all steps of 
pancreatic surgery from one angle with fixed ports. As there is no standard port placement for pancreatic surgeries, it is difficult to accommodate all the above-mentioned angles without due consideration of ergonomics while port placement. Certain laparoscopic pancreatic surgeries have been described on how to perform the surgery from the surgeon's position of standing between the patient's legs ${ }^{[3,4]}$ but we felt that, in this position, the visual and working angle would become fixed. We believed that in a surgery practice like lateral pancreatojejunostomy, the position of the surgeon between the patient's legs would hamper the ergonomics while performing the pancreatojejunal anastomosis, and might not provide proper angle at every surgical step. For prolonged duration of surgical procedures, it is very difficult to operate by standing on one side of the patient with both the right and left working ports on either side of the abdomen (Figure 2a). It becomes very tiring and brings about musculoskeletal disorders to the surgeons as well as their assistant. If the surgeon and his assistant both can operate with working ports on one side of the abdomen, then it will lessen their body strain (Figure $2 \mathbf{b}$ and Figure $2 \mathbf{c}$ ). In order to get a proper angle during complex procedures, there is a need to shuffle the sites of the surgeon, camera, working ports and assistant ports. These procedures require one or two assistant ports for better traction and countertraction. So it needs at least three or four working ports, apart from the camera port. Placement of these various ports needs proper planning to avoid sword-fighting of the instruments inside the abdomen. If there is no standardised protocol for port placement, the operating surgeon has to face the strain and work out the proper port placement every time when undertakes the surgery session. We proposed a standard port placement by taking care of all the above concerns that works for all the laparoscopic pancreatic surgeries.

\section{Proposed standard port placement}

The proposed standard port placements (Figure 3) are: 10-/12-mm umbilical port for telescope (port 0), 12-mm right upper abdominal port in midclavicular line just above the level of umbilicus (port 1), 5-/10-/12-mm left upper abdominal port in midclavicular line just above the level of umbilicus (port 3), 5-mm right (port 2) and left subcostal (port 4) ports just below the costal margin and about $1-2 \mathrm{~cm}$ lateral to the midclavicular line.

Port 0 can be placed supra-, intra- or infraumbilically depending on the site and type of surgery as well as the habitus of the patient. We routinely use a 10-mm 30-degree camera for pancreatic surgery. In selected cases, occasionally it may require a 0 -mm 45-degree camera. Port 1 or 3 or both will be of 12-mm size depending on the type of the surgery and whether endo-GIA stapler will be used or not. It also facilitates the introduction and removal of gauze piece or needle faster without disturbing other ports.

\section{Benefits of the proposed port placement}

With this proposed port placement, the surgeon and assistant can work comfortably by standing on one side of the patient and by using ports on one side of the abdomen (Figure 3). Such proposed port placement provides better ergonomics and maintains all the previously mentioned angles during each step of the surgery. Any sword-fighting inside the abdomen can be avoided. As and when required, the camera port (port 0) can be shuffled between port 1 or 3, which also gives triangulation. As per need, the surgeon and assistant can shuffle their position between each other depending on the stage of the procedure. Either port 1 or port 3 should be placed with a $10-\mathrm{mm}$ or $12-\mathrm{mm}$ size depending on whether endo-GIA stapler is used or not, and on the type of the procedure. This arrangement helps in shuffling the camera position as well as placing the endo-GIA stapler. We generally prefer to place port 1 and 3 with 12-mm size as it gives comfort to insert stapler, sutures and gauze piece very easily without disturbing the telescope and instruments in other ports. It is more appropriate and safer to use all the 12-mm ports in complex surgeries as described by Kendrick in one of his article of total laparoscopic pancreaticoduodenectomy ${ }^{[5]}$. With the proposed port placement, all pancreatic procedures can be easily performed with less difficulty.

\section{Position of the surgeon and his working ports: How to decide}

The two most important factors which decide the positions of the surgeon and the working ports are the site of the surgery and the direction they need to work towards. In general, most of the procedures on the right side of the pancreatic neck can 


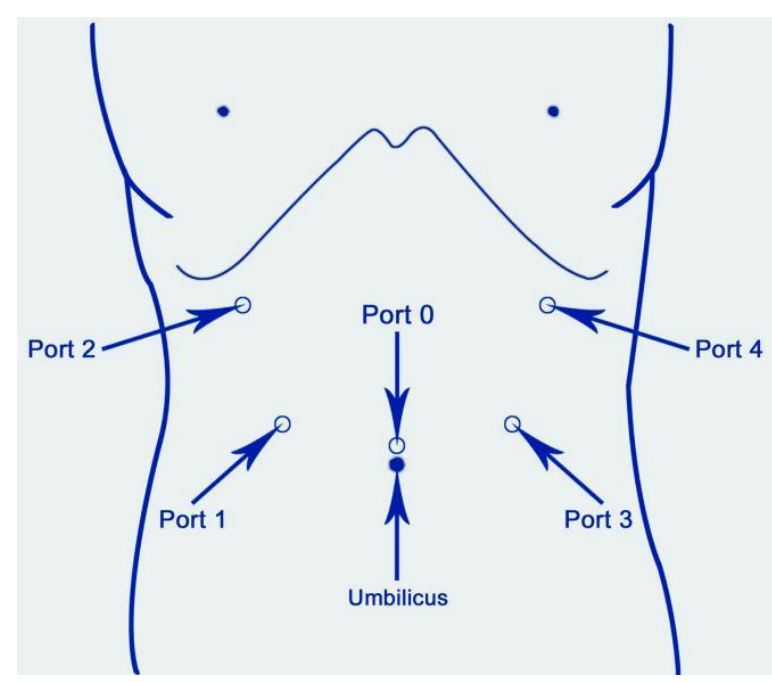

Figure 3. Proposed port placement

be performed while the surgeon is standing on the left side of the patient, using left-sided working ports (ports 3 and 4 , or ports 0 and 4), and procedures on the left side of the pancreatic neck can be operated by standing on the right side of the patient, using right-sided working ports (ports 1 and 2, or ports 0 and 2). For certain parts of the surgery procedure, such as duodenal kocherisation or porta hepatis dissection, the surgery site is on the right side of the pancreatic neck while the direction of the surgery is from the right side to the left side; hence, the surgeon needs to operate by standing on the right side of the patient and uses right-sided working ports.

\section{Steps required during laparoscopic pancreatic surgeries: How to perform with the proposed port placement}

1. Opening the lesser sac

2. Duodenal kocherisation

3. Mobilization of hepatic flexure

4. Mobilization of splenicflexure

5. Transection of first part of the duodenum

6. Gastric transection

7. DJ flexure mobilization

8. Creation of roux loop

9. Gastrojejunostomy

10. Transection of pancreas

11. Uncinate process dissection

12. Hepaticojejunostomy

13. Pancreaticojejunostomy and duodenojejunostomy

14. Lateral pancreatojejunostomy, cystojejunostomy and cystogastrostomy

15. Necrosectomy

\section{Opening the lesser sac}

The surgeon would stand on the right side of the patient while the assistant is on the left side. Both cameras are at port 0 and the surgeon's right- and left-sided working ports would be ports 1 and 2, respectively. Another option is to have camera at port 1 and right- and left-sided working ports both at ports 0 and 2, respectively. One assistant's port, preferably port 3, is sufficient for surgical assistance. The assistant holds the gastrocolic omentum and retracts it, while countertraction would be conducted using the surgeon's left-hand side by holding the stomach near to its greater curve. This manoeuvre lifts up 
the gastrocolic omentum which would be incised with laparoscopic energy device with the surgeon's right hand. With the surgeon standing on the right side of the patient, gastrocolic omentum can be opened from near the antrum to the greater curve up to the angle of need. Beyond the antrum, in order to divide the distal part of the gastrocolic omentum, the surgeon and his assistant need to shuffle their positions to obtain a better angle.

\section{Duodenal kocherisation}

The surgeon stands on the right side of the patient, while the assistant lifts and retracts the duodenum using intestinal graspers from ports 3 and 4 . The surgeon uses ports 1 and 2, or ports 0 and 2, to divide the duodenal attachments. An adequate duodenal kocherisation also needs mobilization of hepatic flexure. Kocherisation can be performed using either scissors or energy devices such as monopolar electrosurgical hook or harmonic scalpel. This procedure can also be performed by standing on the left side of the patient.

\section{Mobilization of hepatic flexure}

The surgeon stands on the left side and uses ports 2 and 4 for dissection. The assistant stands on the right side and uses ports 1 and 3 for retraction of the hepatic flexure. The camera is placed at port 0 . The assistant holds the appendices epiploica (AE) of proximal transverse colon with endoclinch grasper from port 3 and retracts the colon towards the left side of iliac fossa; or holds the appendices epiploica of ascending colon from port 1 and retracts it down to the right side of iliac fossa. This manoeuvre exposes the attachment of hepatic flexure well and the surgeon can divide it using ports 2 and 4 . We prefer to divide this attachment with harmonic scalpel as some of the vessels are always present in this attachment. It is to be noted that hepatic flexure mobilization is easier to perform after the opening of the lesser sac.

\section{Mobilization of splenic flexure}

The surgeon stands on the right side and uses ports 2 and 4 for dissection. The assistant stands on the left side and uses ports 1 and 3 for splenic flexure retraction. The assistant holds AE of distal transverse colon with endoclinch from port 1 and retracts it down to the right side of iliac fossa; or holds $\mathrm{AE}$ of descending colon from port 3 and retracts it down to the left side of iliac fossa. This manoeuvre exposes the attachment of splenic flexure and the surgeon can divide it using ports 2 and 4. This attachment also can be divided comfortably using harmonic scalpel. The mobilization of splenic flexure becomes better after the opening of the lesser sac.

\section{Transection of the first part of the duodenum (D1)}

Transection of the D1 can be done using endo-GIA stapler. The instrument from port 0 gives the best angle for D1 transection. The placement of the camera at port 3, with the surgeon standing on the left side using ports 0 and 4 as the working ports, would be comfortable for this step. The assistant stands on the left side and retracts the stomach and liver using ports 1 and 2 placements.

\section{Gastric transection}

Distal gastric transection is best to be done from port 3, with the surgeon standing on the left side of the patient using ports 3 and 4 as the working ports, while the camera is placed at port 0 and the assistant stands on the right-side using ports 1 and 2 for surgical assistance.

For in-depth proximal gastric transection, the best possible angle is from port 2 . The surgeon stands on the right-side using ports 1 and 2 placements while the camera is placed at port 0 , and the assistant stands on the left side and uses port 3 and 4 positions.

\section{Duodenojejunal (DJ) flexure mobilization}

The surgeon stands on the left side of the patient and uses ports 0 and 4 as the working ports. The telescope goes through 
port 3. The assistant stands on the right-side and uses port 2 for colon retraction and port 1 for jejunal retraction.

\section{Creation of roux loop}

The surgeon stands on the right side and uses port 1 and 2 placements, with the camera placed at port 0 . The surgeon's assistant, who stands on the left side of the patient, retracts the colon up using port 3 and 4 positions. The surgeon runs through the small bowel and identifies the DJ flexure by visualizing the inferior mesenteric vein. The division of the jejunum at the proposed site is carried out using a stapler from port $1(12-\mathrm{mm}$ port $)$. The assistant holds the jejunum at one place with one port while the surgeon holds it at another place using his left-hand-side instrument. By using his right hand, the surgeon fires the stapler through port 1.

\section{Gastrojejunostomy}

Roux-en-Y or loop gastrojejunostomy can be performed in a similar way. The surgeon would stand on the right side and the assistant on the left side, with ports 1 and 2 for the surgeon's position whereas ports 3 and 4 for the assistant. This step needs port 1 of 12-mm size for the endo-GIA stapler insertion. The selection of the site of the stomach for anastomosis is done in order to bring up the loop or roux limb of jejunum near the proposed site of the stomach. The placement of two stay sutures at two edges of the proposed length of anastomosis is done so that the stomach and jejunum will stay together. The left side of stay suture would be held by the assistant (at port 3) while the right side of stay suture would be held by the left-hand side of the surgeon (port 2) in such a way that the proposed gastrojejunostomy stays away and in the straight line with the endoGIA stapler once it is introduced through the surgeon's right-hand side working port (port 1). Two enterotomies are made using harmonic scalpel, upon the insertion of the endo-GIA stapler and its firing. Hemostasis checking is done before the closure of the enterotomy either by intracorporeal suturing or using another stapler. Many surgeons place two sutures at both angles of the anastomosis to reduce the tension over anastomosis.

\section{Transection of pancreas}

For pancreatic transection, the surgeon needs to stand on the right side of the patient. It is preferable to make a tunnel behind the pancreas and encircle the pancreas with umbilical tape that would help in lifting of the pancreas better while transection takes place. The surgeon uses ports 0 and 2 as the working ports with placement of the camera at port 1 . The right-handside instrument through port 0 gives a perfect angle for pancreatic transection at the neck region. It can be transected using various energy sources such as harmonic scalpel, bipolar vessel sealer or monopolar cautery depending on the surgeon's preference.

\section{Uncinate process dissection}

Uncinate dissection is conducted whenever the surgeon is standing on the right side of the patient and the assistant on the left. A camera should be placed at port 1 and the surgeon's working ports are ports 0 and 2. Uncinate dissection is done using ligasure but sometimes for bigger vessels, we use either clip or ligation. This type of dissection can be started from above (top-down approach) or from below (below-upward approach). In top-down approach, 5-mm ligasure at the surgeon's left-hand side enters through port 2 and the right-hand side working port through port 0 . In below-upward approach, 10-mm ligasure at the surgeon's right-hand side enters through port 0 and the left-hand side working port through port 2.

\section{Hepaticojejunostomy (HJ)}

HJ can be done either from the left- or right-side of the patient. We prefer to perform it from the left side using a camera placed at port 3, and ports 0 and 4 as left- and right-sided working ports, respectively. The assistant stands on the right side and uses ports 1 and 2 for surgical assistance. 


\section{Pancreaticojejunostomy and duodenojejunostomy}

Like in pancreatic transection, these steps are done while the surgeon is standing on the right side using a camera placed at port 1 , and ports 0 and 2 as the working ports. The assistant stands on the left side and uses ports 3 and 4 for surgical assistance. Port 0 is at the right-hand side and port 2 is at the left-hand side of the working port for the operating surgeon. As our preferred anastomosis connection part is duct-to-mucosa pancreaticojejunostomy, we first take the posterior layer of anastomosis between pancreatic capsule and seromuscular layer of the jejunum which is in its interrupted manner, following which a small opening is made in the jejunum corresponding to the pancreatic duct wherein the duct-to-mucosa interrupted sutures are placed. Each layer of the suture placement should start from above and end with the lowermost sutures as to not interfere with the vision. After duct-to-mucosa suture placement, the most anterior most sutures are placed between pancreatic capsule and seromuscular layer of the jejunum.

Duodenojejunostomy is done in the similar way as we did in the open procedure using the same ports in ancreaticojejunostomy.

\section{Lateral pancreatojejunostomy (LPJ), cystojejunostomy (CJ), and cystogastrostomy (CG)}

LPJ, CJ and CG can be done in a similar manner while the surgeon is standing on the right side of the patient, with camera at port 1 and the right- and left-sided working ports at ports 0 and 2, respectively. It gives a perfect angle to perform anastomosis across the oblique axis of the pancreas. The assistant uses ports 3 and 4 for surgical assistance.

\section{Pancreatic necrosectomy}

Depending on the site and extent of the necrosis, these ports can be used. The surgeon would stand on the right side and use either ports 0 and 2 or ports 1 and 2 while the camera at either port 0 or 1 . In pancreatic necrosectomy, the camera port should be placed at the supraumbilical location in order to have better access to the cavity. Necrosectomy in the head and right paracolic gutter region requires the surgeon to stand on the left side and to use left-sided ports accordingly.

\section{Application of this port placement for other procedures}

The same port placement can be utilized in gastrectomies, sleeve gastrectomy and esophageal hiatal surgeries.

\section{Summary}

The proposed port placement works comfortably for all laparoscopic pancreatic procedures. It helps shuffle the camera and instrument placements as needed. It is easy to remember, sparing more worries about angulations and ergonomics to think of every time. As the surgeon and the assistant comfortably operate while standing on one side of the table, it gives less stress and better ergonomic convenience. It should be proposed as the standard port placement for these procedures.

\section{Author contributions}

Haribhakti S conceptualized and provided valuable insight from experience, to the making of this manuscript. Mistry JH reviewed the literature and prepared the manuscript.

\section{Conflict of interest}

The authors declared no potential conflict of interest with respect to the research, authorship, and/or publication of this article.

\section{Reference}

1. Correa-Gallego C, Dinkelspiel HE, Sulimanoff I, Fisher S, Viñuela EF, et al. Minimally-invasive vs open pancreaticoduodenectomy: Systematic review and meta- analysis. J Am Coll Surg 2014; 218(1):129-39. doi: 10.1016/j.jamcollsurg.2013.09.005. 
2. Hasukic S. Techniques: Suturing and knot tying in laparoscopy. BH Surgery 2011; 1(1): 53-58

3. Palanivelu C. Laparoscopic pancreatojejunostomy. $1^{\text {st }}$ ed. In: Art of laparoscopic surgery: Textbook and atlas. India: Jaypee Brothers Medical Publishers (P) Ltd; 2007. p. 889-897.

4. Salky B. Distal pancreatectomy. $2^{\text {nd }}$ ed. In: Scott-Conner CEH, (editor). The SAGES manual: Fundamentals of laparoscopy, thoracoscopy, and GI endoscopy. Germany: Springer; 2006. p. 393-399.

5. Kendrick ML, Cusati D. Total laparoscopic pancreaticoduodenectomy: Feasibility and outcome in an early experience. Arch Surg 2010; 145(1): 9-23. doi: 10.1001/archsurg.2009.243. 